\title{
Progress and Challenges of Reference Standard and Its New Form: Digital Reference Standard
}

\author{
Qingjun Wang1*, Lei Sun ${ }^{1,2}$, Feng Liu², Hongyu Jin'1, Jiandong Yu1, Zhong Dai', \\ Shuangcheng Ma1\# \\ ${ }^{1}$ National Institute for Food and Drug Control, Beijing, China \\ ${ }^{2}$ Xinjiang Institute for Food and Drug Control, Urumqi, China \\ Email: zjswqj@163.com, dasunlei@sina.com, "masc@nifdc.org.cn
}

Received 27 May 2016; accepted 27 June 2016; published 30 June 2016

Copyright (C) 2016 by authors and Scientific Research Publishing Inc.

This work is licensed under the Creative Commons Attribution-NonCommercial International License (CC BY-NC).

http://creativecommons.org/licenses/by-nc/4.0/

(c) () (9) Open Access

\begin{abstract}
Number and quantities of reference standards (RS) needed for the quality control of drugs are increasing, bringing great pressure to the calibration and using company. This manuscript summarized the four generations for the development of $\mathrm{RS}$ including physical RS, paper atlas, substitute $\mathrm{RS}$ and electronic databases. The advantages and disadvantages of each generation were summarized. The concept of digital RS (DRS) was proposed based on this, and summed up the definition, advantages, and technical architecture of DRS. The 10 characteristics of five aspects of the DRS were discussed including digital, multi-dimension, big data, cloud computing, internet, internet of things, sharing, multi-terminal, intelligence, and compliance certification. Then, the necessity of its formation and application in the medicine holistic quality control of internet plus era was discussed in this manuscript.
\end{abstract}

\section{Keywords}

Digital Reference Standards, Herbal Reference Standards, Extractive Reference Standards, Substitute Reference Standards, Holistic Quality Control, Internet Plus, Big Data, Database

\section{Introduction}

Quality control is an important part of drug development and production including character, identification, test,

\footnotetext{
*The first two authors contributed equally.

"Corresponding author.
}

How to cite this paper: Wang, Q.J., Sun, L., Liu, F., Jin, H.Y., Yu, J.D., Dai, Z. and Ma, S.C. (2016) Progress and Challenges of Reference Standard and Its New Form: Digital Reference Standard. Chinese Medicine, 7, 77-91. 
and assay. These items cannot be carried out without reference standard (RS), which has a definite characteristic or value, used for determining the potency, quality or purity of a drug, and also used for correcting test apparatus and method. These standards include RS for the test of Traditional Chinese Medicine (TCM), chemicals, biological products, and pharmaceutical excipients (PE) [1]-[3]. At present, types and quantities of RS needed for the quality control of drugs are increasing, bringing great pressure to the calibration and using company. For example, multi-component analysis of TCM requires several chemical RS (CRS), but the RS are difficult to purify, with high prices, unstable, toxic or have security risk. To carry out the test of the related substance of chemical drugs requires several impurity RS, but the synthesis of them is difficult with high cost of usage. There are many kinds of pesticide RS of high toxic, unstable, and even many varieties have been discontinued [1]-[11].

These problems greatly affect the provision and use of RS. To solve these problems, researchers have proposed and developed a variety of RS as follows: 1) integration of the data in the literature to draw a set of paper maps for reference (but this set is not convenient to query and slow updates, and sharing degree is not high) [12]-[16]; 2) substitute RS, often using one RS for qualitative analysis (using the relative retention time method) and multiple components for quantitative analysis (using the relative correction factor method). This method is often used in the testing of related substances in chemical medicine and multi-component analysis of TCM [17][34]; and 3) the paper map combining with the computer technology to form an electronic database (offline or online version), which can be shared and referred with the data. However, the current database intelligent degree is not high. Most of them are the isolated database in fragmentation. Data format is not unified. The internet function is imperfect. These reduce the utilization of achievements in scientific researches and hinder the international collaboration and sharing information [35]-[53].

In this manuscript, the development of RS was reviewed. The problems present in the forms of existing RS such as physical and alternative RS, paper map set, and electronic database were discussed. Based on this and combined with internet plus and big data technology, the concept, related characteristics, and technical architecture of digital RS (DRS) were put forward. Digitizing the drug RS not only can reduce the preparation and calibration of physical RS, the waste of resource and environmental pollution to save the cost, but also can provide more comprehensive and interrelated information in the systematic and standardized form to achieve intelligent search, big data storage, and internet sharing purposes [54]. DRS provide a new idea and method for the development of RS and quality control of drug.

\section{The Development of the RS}

\subsection{Physical RS}

Physical RS is RS in kind and is used as reference in most of the current items in drug quality control.

\subsection{1. "Chinese Pharmacopoeia (ChP)" Supporting Physical RS}

RS for the test of Chinese medicine mean RS that used in verification of medicinal herbs (including prepared), extract, and Chinese Patent Medicines including CRS, herbal RS (HRS), extractive RS (ERS), and residual RS (Figure 1). ChP first received the CRS of Chinese medicine in version 1985, for example, Eugenol RS is used in the identification of medicinal herbs clove [4]. HRS refers to the originally clear, with accurate parts, and high quality Chinese medicinal herb powder [2]. For example, ChP first used the rhubarb HRS in the identification of rhubarb in version 1985 [4]. HRS is China's first and characteristics of RS form. It retains all the ingredients of the herb and the powder characteristics with wide usage. ERS refers to the mixture containing a variety of main effective components or indicators prepared by the specific extraction process [2]. For example, ChP first used rose essential oil in the identification of xianlianpian in version 2005 [5]. In addition, residue RS and work substance are included, and are mainly used for heavy metals, pesticides, and aflatoxin test. For example, aflatoxin mixed standard and 22 kinds of organic chlorine mixed control solutions [6] are used to test aflatoxin for Chenpi, Pang Dahai in ChP first chapter of version 2015.

RS for chemical drugs refer to national RS having single component, composition or mixed composition that used to test antibiotics, chemical, biochemical, and radioactive drugs as well as instrument calibration.

Figure 2 is the statistician of RS for TCM and pharmaceutical chemicals (PC) in calendar edition of ChP. Among them, HRS and CRS for TCM part refers to the review by Lingyun Ma et al. [7]. PC part refers to calendar edition of pharmacopoeia appendix. The number and types of ChP RS in version 2015 are shown (Figure $3)$. 


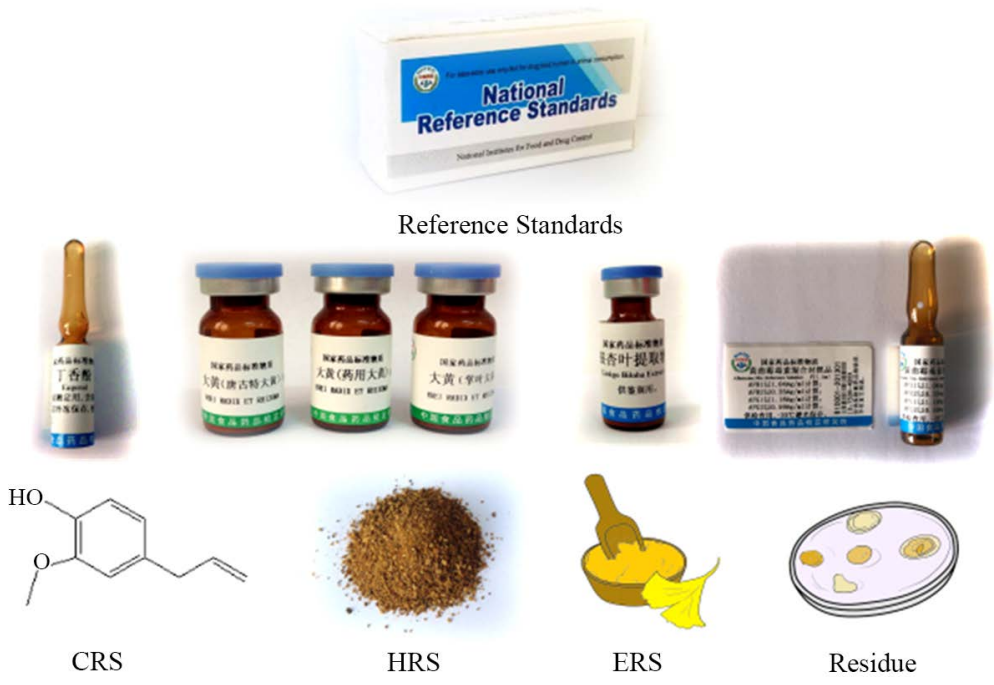

Figure 1. Types of reference standards used in Traditional Chinese Medicine.

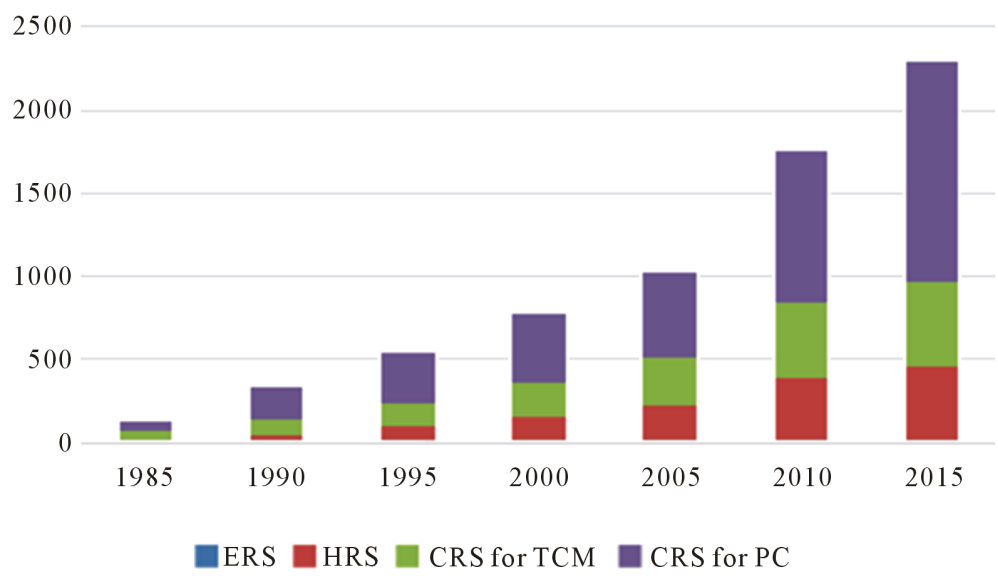

Figure 2. The number and growth of ChP reference standards (excluding BRS). (X coordinate: Year; Y coordinate: Number of species).

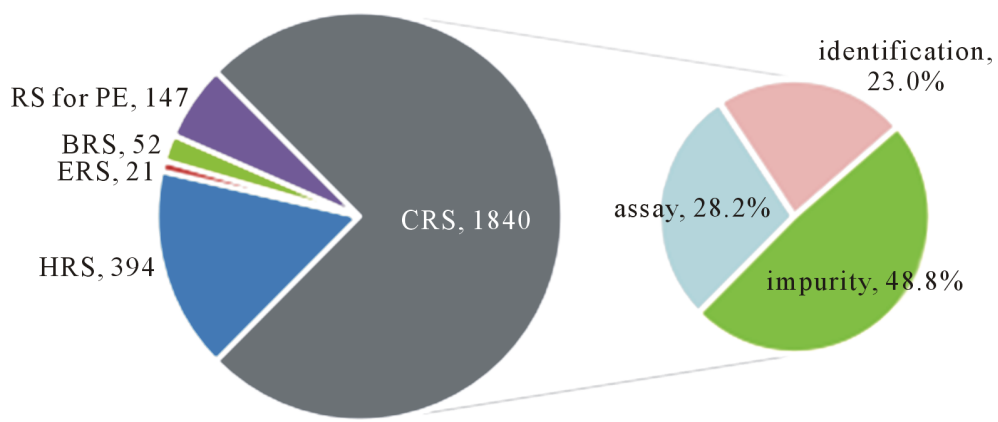

Figure 3. Number and types of ChP reference standard (2015).

The biological reference standard (BRS) including vaccines, antibodies, and serum diagnostic reagents, et al. refers to that extracted and purified from an organism and used in the determination of biological potency, activity, concentration, and content and other related testing for biological products. Diphtheria toxin was officially recognized as the first international BRS in 1922 [55]. Fifty-two types of BRS were collected in 2015 version of ChP [3]. Because of earlier pharmacopoeia version had not listed catalogue varieties of BRS or verification of 
biological products all referred to "Chinese Requirements for Biological Products” [8]. There is no statistics here.

2015 edition of ChP first separated the PE to inductive branch. Additionally, a large amount of RS was used for identification, test, and determination of PE quality control. For example, lactide-co-glycolide (PLGA) (50:50) (for injection) used polystyrene molecular weight control products for the test of molecular weight distributions [3].

\subsubsection{RS Used in Foreign Pharmacopoeia}

Mainly responsible for the establishment and management of drug RS internationally are the European Directorate for Quality Medicines (EDQM), United States Pharmacopoeia (USP), Laboratory of the Government Chemist (LGC), and Public Book Association of Japan.

Around the world, more than 50 countries use the European Pharmacopoeia (EP) RS including CRS (for natural and chemical medicine), HRS, ERS, BRS, and standard spectra (non-physical RS). Among them, 2422 kinds are CRS. They are mainly used for content determination, impurity, and system suitability test, and peak location (Figure 4). Other uses including identification, microbial content determination, chromatographic behavior tests and verification, nuclear magnetic resonance (NMR), impurity test, and chemical identification [9].

Early American RS used were mainly biological complex substances such as pepsin (1936) and cod liver oil (1942). In the past few decades, analysis methods gradually changed from direct to the instrument analysis. Number of USP RS has increased significantly (Figure 5). This shows that the demand for RS is enormous in era of instrument analysis. In USP current version (USP 38), six kinds of RS are collected including CRS, HRS, ERS, BRS, residual solvent RS, and standard micrographs. Among them, 3427 kinds are CRS. They are mainly used for content determination, test for related substances and impurities, identification, and system suitability test after classified refinement per the use (Figure 6). Other uses including melting point determination and resolution test [10] [11].

It has been noticed that the EP collected 127 standard spectrums of 127 kinds of substances, and the USP collected six standard micrographs. This means that the drug multi-dimensional information would be used as RS in quality control.

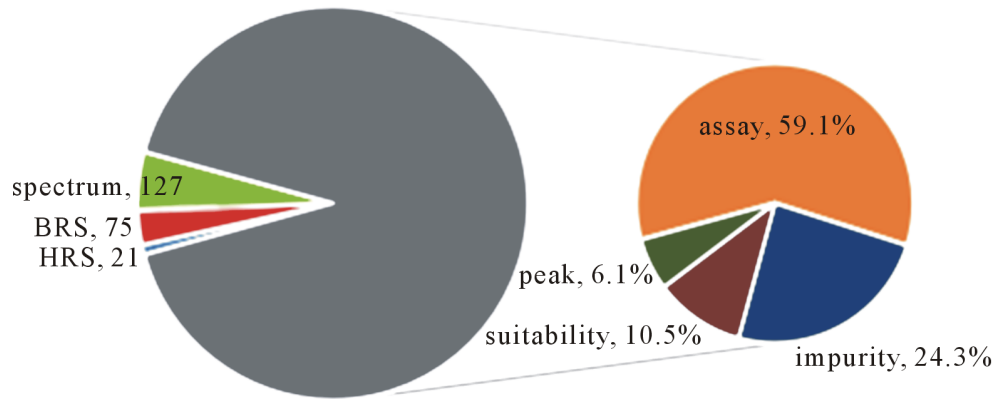

Figure 4. Number and types of EP reference standards (2015).

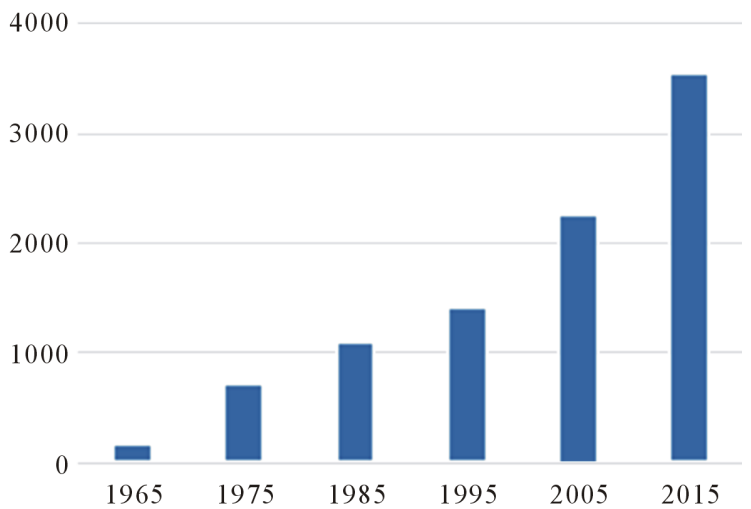

Figure 5. Number and increase of USP reference standards. (X coordinate: Year; Y coordinate: Number of species). 


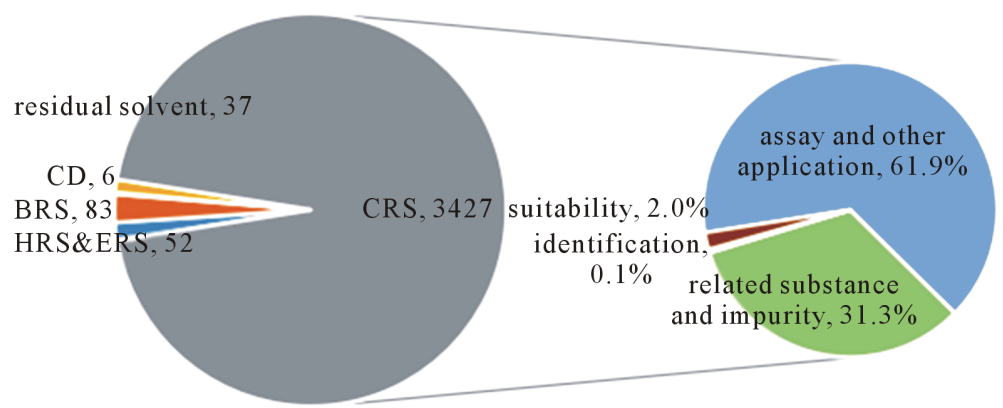

Figure 6. Number and types of USP reference standards (2015).

In summary, the development of RS for various countries has the following characteristics: 1) variety of different characteristics. EP and USP are mainly controlled by CRS; whereas China has characteristic HRS and 16\% of RS in ChP, relatively higher than others. 2) The number is gradually increasing. At present, each country has 2000 to 3500 kinds of RS. Owing to the different entries of drugs in different countries, and different definition of several types of RS, there is no comparability between the numbers of different countries' pharmacopoeia RS. From above analysis, it has been noticed that quantity oriented driving mode is challenged due to difficulty in synthesis and preparation, and high cost of usage. Hence, the appearance of new mode for RS is in urgent need.

\subsection{Paper Atlas}

Some characteristic data and information of a series of physical RS are compiled to be a paper atlas. Though these maps are tool books, it can be used for comparison and reference, so with the attributes of RS.

In some countries or regions directly compiled the maps into text. For example, "Hong Kong Chinese Materia Medica Standards" collected the characteristic and microscopic structure of corresponding medicinal herbs in each theory (Figure 7) [12]. Similarly, EP compiled standard spectra of 127 kinds of substances. USP also compiled six standard micrographs. China often adopts the way of publishing pharmacopoeia matching books. For example, ChP Commission publishes the "Atlas of Infrared Spectra of drugs", "Atlas of TLC Color pictures of Chinese Crude Drugs for Chinese Pharmacopoeia”, and "An Illustrated Handbook on Microscopic Identification of Chinese Crude Drugs for Chinese Pharmacopoeia” [13]-[15]. In addition, there are several third party published map atlas, tool books, and documents. For example, the Sadtler research laboratory in US has published a large spectral atlas "Sadtler Reference Spectra Collections" since 1967. It has been widely used in the international including standard IR (two sets of prism and grating), ultra violet (UV), and NMR hydrogen spectrum. From 1976, began to collect NMR carbon spectrum [16].

The paper atlas and its development have the following characteristics: 1) easy to use, cost effective, and the map is more intuitive and specific; and 2) mainly used for qualitative analysis, but quantitative analysis is its short board. 3) The paper atlas is inconvenient for carrying and query. On the other hand, update is slow. 4) The paper atlas is adopted widely in many countries. Its types and numbers continue to increase. However, still it has faced with challenge of digitization.

\subsection{Substitute RS}

Substitute RS refers to a method for qualitative and quantitative determination of another one or more components to be measured by one or a few physical RS using several constant Eigen values and algorithms. In consideration of the ERS can replace several CRS [17], therefore classified as a substitute RS.

At present, the widely accepted qualitative method is the relative retention time, and the quantitative method is the relative correction factor. The above methods have been used in pharmacopoeia of many countries, and widely used in related substance test of PC, Chinese medicine feature, fingerprint map, and multi-component determination [18]-[29].

In addition, qualitative analysis include new methods such as linear calibration using two RS (LCTRS) [30], ERS, and UV-visible spectrum [31] [32], and mass spectrometry (MS). The LCTRS method is shown in Figure 8 [30]. The standard retention time is used as a benchmark value of prediction. Linear relationship is used as a basic algorithm to predict the retention time. These predictions have a great practical value and application 


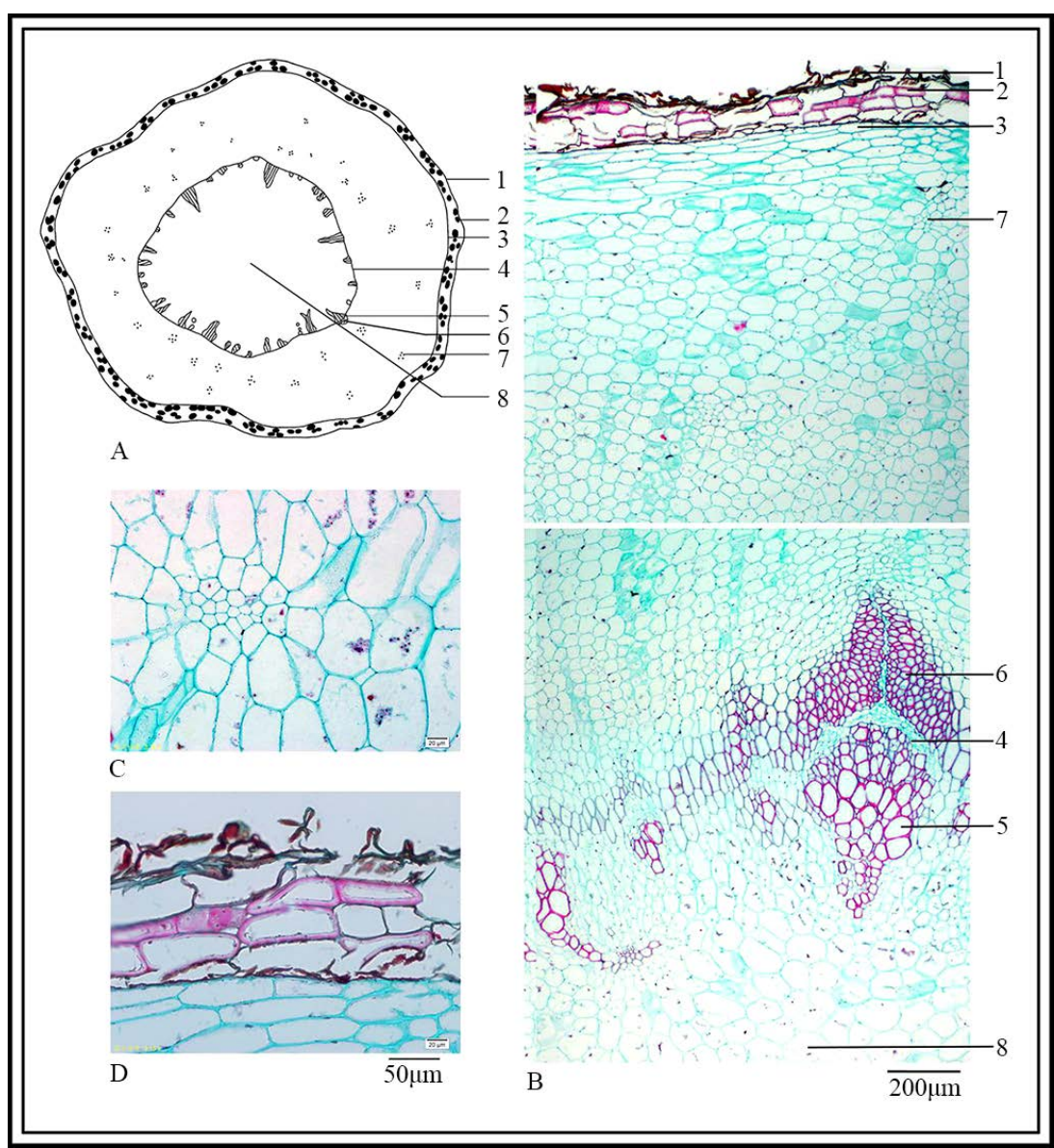

Figure 7. Microscopic characteristics example of seventh Hong Kong Chinese Material Medicinal Standards (Aconiti Kusnezoffii Radix).
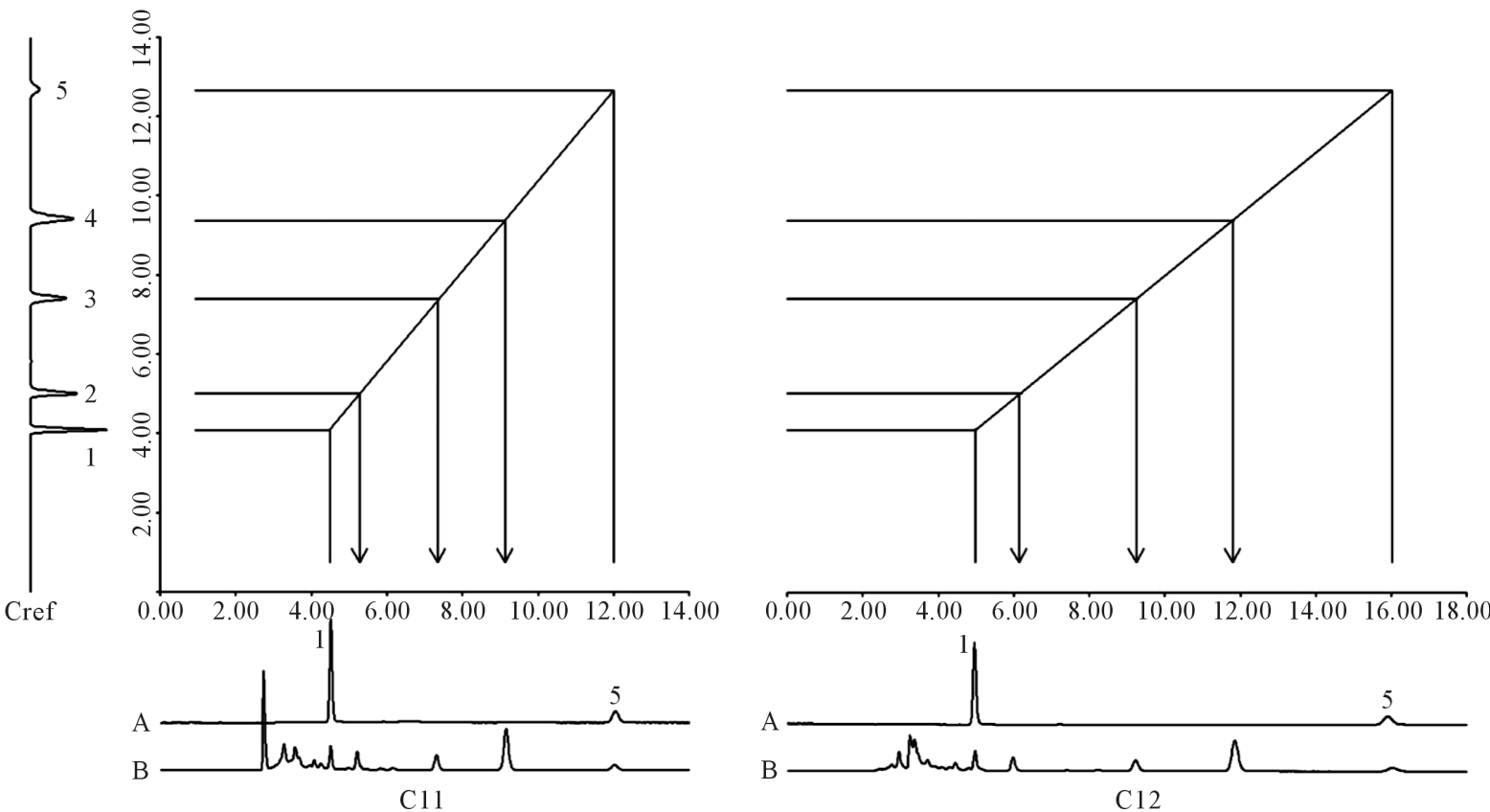

Figure 8. Schematic diagram of LCTRS method. 
prospects. Quantitative methods include ERS and quantitative structure-ion intensity relationship strategy (QSIIR, suitable for MS detector) [33] [34]. QSIIR is also a new method, which provides a new way of thinking for alternative RS by establishing the quantitative linear relationship between the composition structure and relative ion intensity factor.

Alternative RS method has the following characteristics: 1) reduce the use of physical RS and testing cost; 2) there are some new technologies and methods, but only the relative retention time, correction factor, and ERS method are collected into the pharmacopoeia, and the practicability of other methods remains to be explored; 3) the accuracy and repeatability of relative retention time method collected from pharmacopoeia remain to be improved and need further research; and 4) paper atlas mainly provides qualitative features, this method can also provide quantitative characteristics.

Alternative RS is an effective way to solve the problem of physical RS, and it is worth popularizing. The future development direction for qualitative aspects is as follows: 1) the characteristic value and the algorithm are written into software. 2) Varieties of technique are combined.

\subsection{Electronic Database}

The electronic database can be regarded as the digital form of the paper atlas including the early database with less data, simple function and new one with more information and functions.

Early database includes organic compounds MS information MassBank [35], plant metabolites chromatography (GC)/MS information database: Golm metabolome database [36], and chromatographic column recommendation system for antibiotics in 2005 edition ChP antibiotic.CN [37]. With the deepening of the research work, analysts integrated databases with internal links. Later, the new database has many advantages in functional diversity, data storage, and quantity comparing to the early database (Table 1). The quantity of data of the two types of databases is just a relative amount. Some publicly available database and their functions are listed in Table 2.

Electronic database is a software form of RS, which has following characteristics: 1) retains the intuition of comparison of image based paper atlas, also has the advantage of automation, and query convenient. 2) Existing problems of uniform data format, less data dimension, not great enough data quantity, weak online sharing, and intelligent computing function. It is still in the primary stage of digitization of RS.

\section{DRS}

\subsection{Definition of DRS}

The concept of DRS for pharmaceutical analysis is first proposed by the author in the view of the existing problems and shortcomings, combining with the current development trend of information, intelligence, and internet plus in the world. DRS are a kind of digital, data, internet, and intelligent form of RS. DRS is used for the qualitative and quantitative test of drugs instead of RS. It is a multi-dimensional database and intelligent computing software, especially online sharing platform, and conforms to certain specification.

\subsection{Characteristics of DRS}

Compared with the current compound database or software, the DRS have more abundant connotation and function. The 10 characteristics of five aspects of the DRS are discussed as follows: first is information, which is the inner form of DRS; second one is cloud, which is the database of DRS; third is the network, which is the communication bridge between DRS; fourth is the end, which is carrier interaction of DRS; and fifth is the norm, which is the quality assurance of DRS. The following details of each feature are combined in Figure 9.

Table 1. Comparison of the characteristics of the new type and the early database.

\begin{tabular}{cccc} 
Database & Data storage mode & Database functions & Data types \\
\hline Early stage & $\begin{array}{c}\text { Mostly CD rom or remote } \\
\text { connection machine }\end{array}$ & Simple query & Single \\
New type & Web & $\begin{array}{c}\text { Including query, } \\
\text { comparison, calculation, } \\
\text { and other functions }\end{array}$ & $\begin{array}{c}\text { Related multi-dimensional } \\
\text { information, but mostly } \\
\text { in literature type, and } \\
\text { inconvenient for } \\
\text { statistical analysis }\end{array}$ \\
\hline
\end{tabular}


Table 2. Examples for new databases.

\begin{tabular}{|c|c|c|}
\hline Database name & Data contents & Functions \\
\hline Sadtler IR [38] & $\begin{array}{l}\text { Collection of 259,000 pieces of IR spectrum including } \\
\text { polymers, pure organic compounds, industrial chemicals, } \\
\text { dye pigments, drugs and illegal narcotic drugs, fiber and } \\
\text { textiles, spices and flavor, food additives, pesticides and } \\
\text { agricultural products, monomer, important pollutants, } \\
\text { multi alcohols, and organic silicon. }\end{array}$ & $\begin{array}{l}\text { Spectrum retrieval, at the same time, } \\
\text { display information on composition, } \\
\text { chemical and physical properties, sample } \\
\text { sources, classification, and structure } \\
\text { of the compound. }\end{array}$ \\
\hline $\begin{array}{l}\text { Madison metabolomics } \\
\text { consortium [39] }\end{array}$ & $\begin{array}{l}\text { NMR, MS, molecular weight of isotope isomer, chemical } \\
\text { shift of experience and calculated values, the name, } \\
\text { structure, physical, and chemical properties of the } \\
\text { metabolites for }>19,700 \text { compounds. }\end{array}$ & $\begin{array}{l}\text { Different ways of retrieval such as } \\
\text { structure, NMR, MS, data statistics, } \\
\text { and site related links. }\end{array}$ \\
\hline NCI $[40]$ & Collection of 250,250 compounds for query. & $\begin{array}{l}\text { Different ways of retrieval such as name, } \\
\text { CAS number, and molecular mass range, } \\
\text { anti-HIV activity, LogP, and compound } \\
\text { structure, and multi format output. }\end{array}$ \\
\hline EDCs $[41]$ & $\begin{array}{l}\text { More than } 600 \text { kinds of 3-dimentional structure of } \\
\text { endocrine disrupting substances including molecular } \\
\text { name, structural characteristics, drug efficacy, and } \\
\text { toxicology information. }\end{array}$ & $\begin{array}{l}\text { Multi ways and similarity retrieval such } \\
\text { as 2, 3-dimentional molecular structure } \\
\text { download, statistical analysis diagram, } \\
\text { and related links. }\end{array}$ \\
\hline $\begin{array}{l}\text { NIST mass spectral } \\
\text { library [42] }\end{array}$ & Collection of 64,000 pieces of mass spectra & $\begin{array}{l}\text { Mass data query, comparison, } \\
\text { and analysis of spectra. }\end{array}$ \\
\hline MetaCYC [43] & $\begin{array}{l}\text { Metabolic pathway database including genes, } \\
\text { proteins, compounds, reaction, pathways, } \\
\text { and other information. }\end{array}$ & $\begin{array}{l}\text { Basic, advanced, and cross biological } \\
\text { search, data comparison and analysis, } \\
\text { statistical analysis, and other functions. }\end{array}$ \\
\hline $\begin{array}{l}\text { Waters UNIFI scientific } \\
\text { information system [44] }\end{array}$ & $\begin{array}{c}\text { Chromatography, MS, data management and laboratory } \\
\text { work flow integration platform including seven solution, } \\
\text { biopharmaceutical, forensic toxicology screening, } \\
\text { identification of metabolites, natural products, } \\
\text { pesticide screening, regulatory compliance } \\
\text { bioanalysis, and screening platform. }\end{array}$ & $\begin{array}{l}\text { Including data collection, processing, } \\
\text { browsing, report generation, and } \\
\text { configurable regulatory compliance tool. }\end{array}$ \\
\hline $\begin{array}{l}\text { Aglient Technologies GC/MS, } \\
\text { LC/MS chemical synthetic } \\
\text { drug storehouse [45] }\end{array}$ & $\begin{array}{l}\text { GC/MS library including }>300 \text { kinds of chemical synthetic } \\
\text { drugs and its metabolite spectrum diagram, >500 kinds of } \\
\text { chemical synthetic drug recent metabolites. GC/MS/MS } \\
\text { MRM MassHunter database in EI or CI mode, chemical } \\
\text { synthetic drug collection. The LC/MS library contains } \\
\text { the forensic/toxicology precision quality database of } \\
9000 \text { compounds, and accurate MS/MS spectrum } \\
\text { library of } 3000 \text { compounds. }\end{array}$ & $\begin{array}{l}\text { Compatible with ChemStation and } \\
\text { MassHunter software using Agilent personal } \\
\text { compounds database (PCD) or PCD and } \\
\text { library for fast implementation screening } \\
\text { method, and screening the new chemical } \\
\text { synthetic drugs. }\end{array}$ \\
\hline Thermo Fisher spectrum [46] & $\begin{array}{l}\text { Including Aldrich, forensic analysis, polymer, } \\
\text { Raman, sigma biochemical, gas phase, } \\
\text { and impurity spectrum. }\end{array}$ & $\begin{array}{l}\text { A large number of spectral information } \\
\text { of compounds is collected for query. }\end{array}$ \\
\hline $\begin{array}{l}\text { Metabolic enzymes in yeast } \\
\text { LC/MS/MS MRM [47] }\end{array}$ & $\begin{array}{l}\text { Database has } 3584 \text { pairs of MRM ions including } 13 \mathrm{C} \\
\text { labeled isotope, containing the } 1792 \text { pairs of MRM ions } \\
\text { of } 498 \text { peptides produced by enzymatic hydrolysis } \\
\text { from } 228 \text { enzymes of budding yeast. }\end{array}$ & $\begin{array}{l}\text { Can analyze the } 228 \text { kinds of primary } \\
\text { metabolism related enzyme from enzymatic } \\
\text { hydrolysis of the pancreatic polypeptide } \\
\text { of budding yeast. }\end{array}$ \\
\hline $\begin{array}{l}\text { Smart MRM database of } \\
\text { poison version [48] }\end{array}$ & $\begin{array}{l}\text { This database covers } 1200 \text { MRM channels of } 201 \text { forensic } \\
\text { and toxicology related compounds. These compounds } \\
\text { include drug abuse, psychotropic drugs, pharmaceuticals, } \\
\text { and pesticides. The database collected retention index } \\
\text { of compound, MRM channel, collision energy, } \\
\text { and ion ratio correlation information. }\end{array}$ & $\begin{array}{l}\text { High selectivity detection based on MRM, } \\
\text { automatically create best MRM method, } \\
\text { and collect SCAN/MRM modes at the } \\
\text { same time combined with "GC/MS } \\
\text { database of poison version”. }\end{array}$ \\
\hline
\end{tabular}




\section{Continued}

ChEBI [49]

Dictionary of natural products [50]

Chem-TCM [51]

New Drug Oriented Development System of Chinese Medicine [52]

Chinese Medicinal Meterial Images Database [53]
A large number of active small molecule compounds including atoms, molecules, ions, ion pair free radical, and free radical ion.

Chemical, physical, and biological properties, system nomenclature and generic names, reference, structure diagrams, and related forms of compound molecules.

More than 12,000 compound records including $>350$ kinds of medicinal plants, containing information of chemical, botany, molecular targets, category of TCM, and expected activity.

Two-thousand disease names, 180,000 prescriptions of TCM, 9000 kinds of TCM, 25,000 (15,520 structures) compounds, and $>1700$ target proteins of 147 kinds of diseases.

Including characteristics and partial microscopic identification of $>420$ kinds of commonly used TCM, and detailed description and comparison are done in a graphical way. At the same time, information as the sources of medicinal plants, the main origin, character, quality characteristics, and the effectiveness are recorded.
Basic and advanced search, multi format data download, and a variety of analysis tools.

Multi-channel retrieval such as chemical name, molecular formula, CAS number, whole paper, melting, and boiling point.

Multi-channel retrieval, sort, result filter, and database subset creation.

Retrieval and a variety of statistical analysis.

Supporting many types of retrieval as the medicine, Latin, English, the original plant and animal name, and effectiveness.
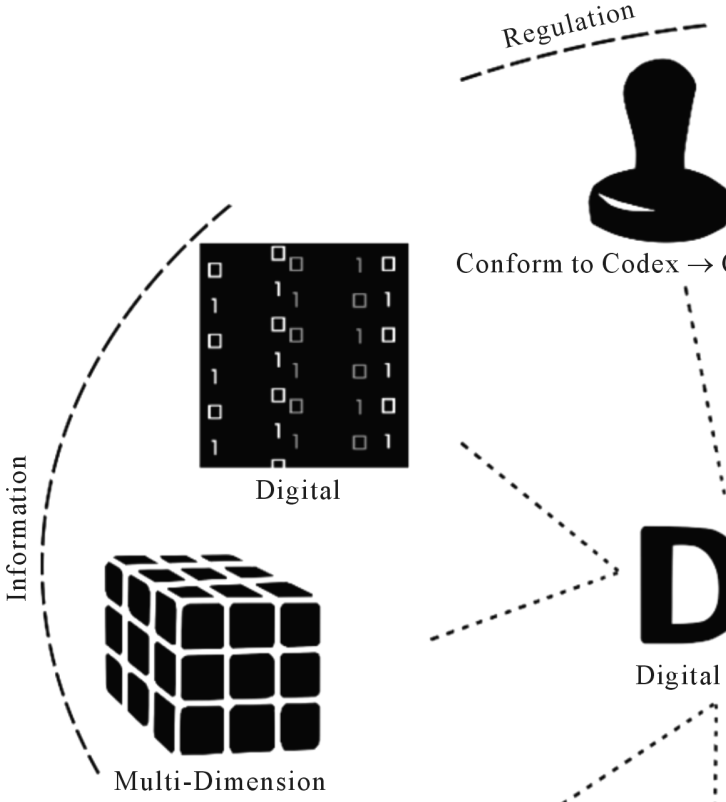

Conform to Codex $\rightarrow$ Certification

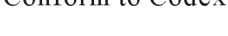

:
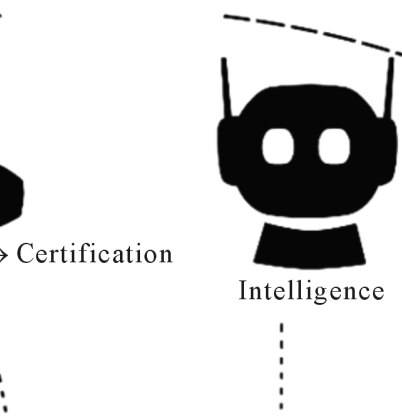

Intelligence
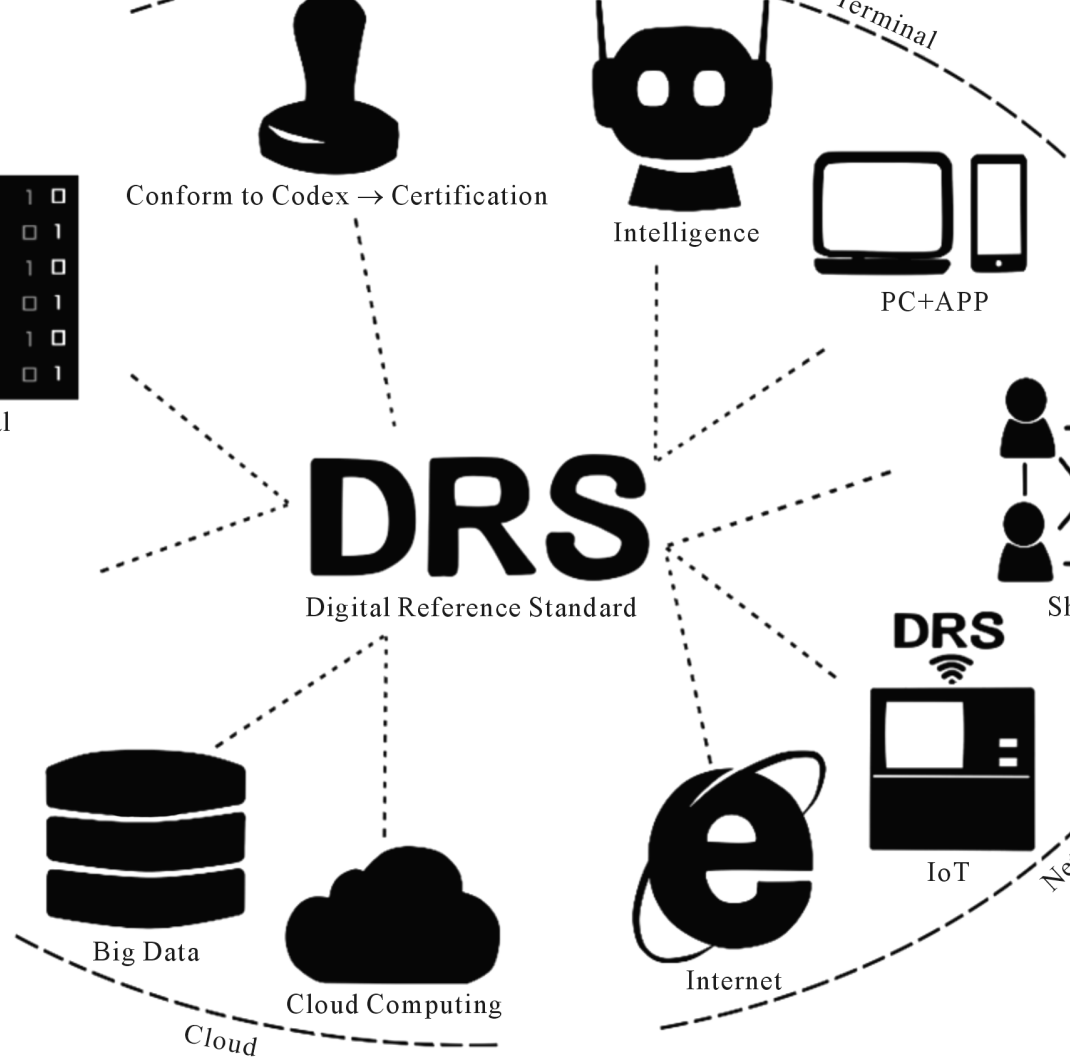

$$
\mathrm{PC}+\mathrm{APP}
$$

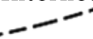

Figure 9. Characteristic of digital reference standards. 
1) Digital

Digital is the inevitable development trend of RS, is also the most basic feature of DRS.

2) Multi-dimension

Different dimension refers to different physical, chemical, and biological characteristics contained of physical RS in the DRS or information reflecting these features. The former is called A class dimension, the latter is called B class dimension. The molecular structure, melting point, optical rotation value, and IR spectrum information of chemical monomer belongs to A class dimension. Speckle characteristics and other information of HRS in different developing conditions are classified into B class dimension. This information is the reflection of ingredients and composition characteristics of this HRS in different conditions. DRS have a multi-dimensional structure. A high degree of internal connection among different dimension of data can be established by information and computer science technology, therefore forming a comprehensive system of information network.

3) Big data

Currently, drug standard or electronic database usually give strict method and unique result for reference used for one variety. This is a single (or small) sample collected "standard information" database. This is more appropriate for easy standardized analysis test or easy standardized instrument such as IR spectrum database or standard National Institute of Standards and Technology library for GC/MS.

But some test results are affected by many complex factors. TLC identification method, for example, due to the difficult to accurately control and repeat of the thin layer plate, temperature, humidity, and the expansion condition "standard retention frequency value" specified in quality standard is difficult to repeat. Supporting TLC map is the only results of the individual laboratory and weakens its reference role. The author believed that the concept of big data could be used, that is, not to seek a standard value, but to collect the information (photos, sketch map, and other information) in different conditions (in a certain range) into database. Similarly, high performance liquid chromatography (HPLC), liquid chromatography-MS, and other databases of the corresponding RS can be established based on this method.

In addition to preserve the feature information, the big data can record the user login and other data. The data can be used for user portrait, version improvement, and intelligent recommendation.

4) Cloud computing

When DRS are really built and placed into use, would face huge amounts of data and search request. Therefore, the cloud computing is an inevitable choice. But this technique needs to pay attention to the problem of data security.

5) Internet

DRS is not a localized program, but a server and client system are connected through the internet. This would be conducive to immediate use and rapid update for the system as well as the technical support of the sharing and intelligence of DRS.

6) Internet of things

Through a uniform interface and data interchange format, DRS can connect to a variety of instrument analysis such as laboratory information management system, electronic laboratory notebook, and electronic quality management system and transfer, and process raw data directly.

7) Sharing

To reduce the repeated research, improve the utilization of research results, DRS need to introduce information sharing mechanism. The user can apply it for deletion or amendment, but need to review and certify the sharing information. In addition, corresponding incentive sharing mechanisms are also need to be established.

8) Personal computer and application version

Mobile phones, tablet computers, and other mobile devices have gone deep into every corner of the modern life and work. To adapt to the technology of the trend, the DRS also has two versions namely the personal computer software and the mobile application.

9) Intelligence

The current digital projects are often a browsing database with simple query function. The author thought that the real DRS should be a program of combination of database and artificial intelligence (especially machine learning techniques). It has a powerful search engine, pattern recognition, and other computing functions. The next step may use virtual and augmented reality technology. This would play a great role in the display of characteristics of TCM herbs. 
10) Certification and compliance

The DRS must conform to the requirements of the regulations as physical RS. Hence, it is necessary to review the data uploaded by the qualified institutions per relevant standards, to ensure the authenticity, reliability, and accuracy of the data.

\subsection{Design of DRS}

DRS include big data, intelligent analysis, platform client, identity authentication, and system service subsystems. The system design is shown in Figure 10.

1) Big data subsystem. The subsystem is the core information source of DRS application platform, comprising all the spectroscopies, MS and NMR spectroscopy database, relevant TLC, HPLC, and GC sample graph database as well as Chinese crude drug information database of each RS.

The subsystem design philosophy fully embodies the idea of big data: the information of one RS would include data results from different sources and equipment brand. With the rolling accumulation of sample data, hit rate of database searching and reliability of results would be increased to a maximum extent.

2) Intelligent analysis subsystem. The subsystem is the core application layer of DRS application platform. It is designed for the cross-libraries joint matching of compound spectra of user input (similarity retrieval), and further gives qualitative identification in sample spectra (prediction of the retention time). The new technology includes spectra column positive and negative list based on big data, "LCTRS", retention time correction method. To realize the top design requirements of daily test work using DRS instead of TRS.

The subsystem would use artificial intelligence technology, through a variety of instruments analysis data pre-treatment techniques and stoichiometry algorithms, to match, compare, and analyze the data and spectrum in

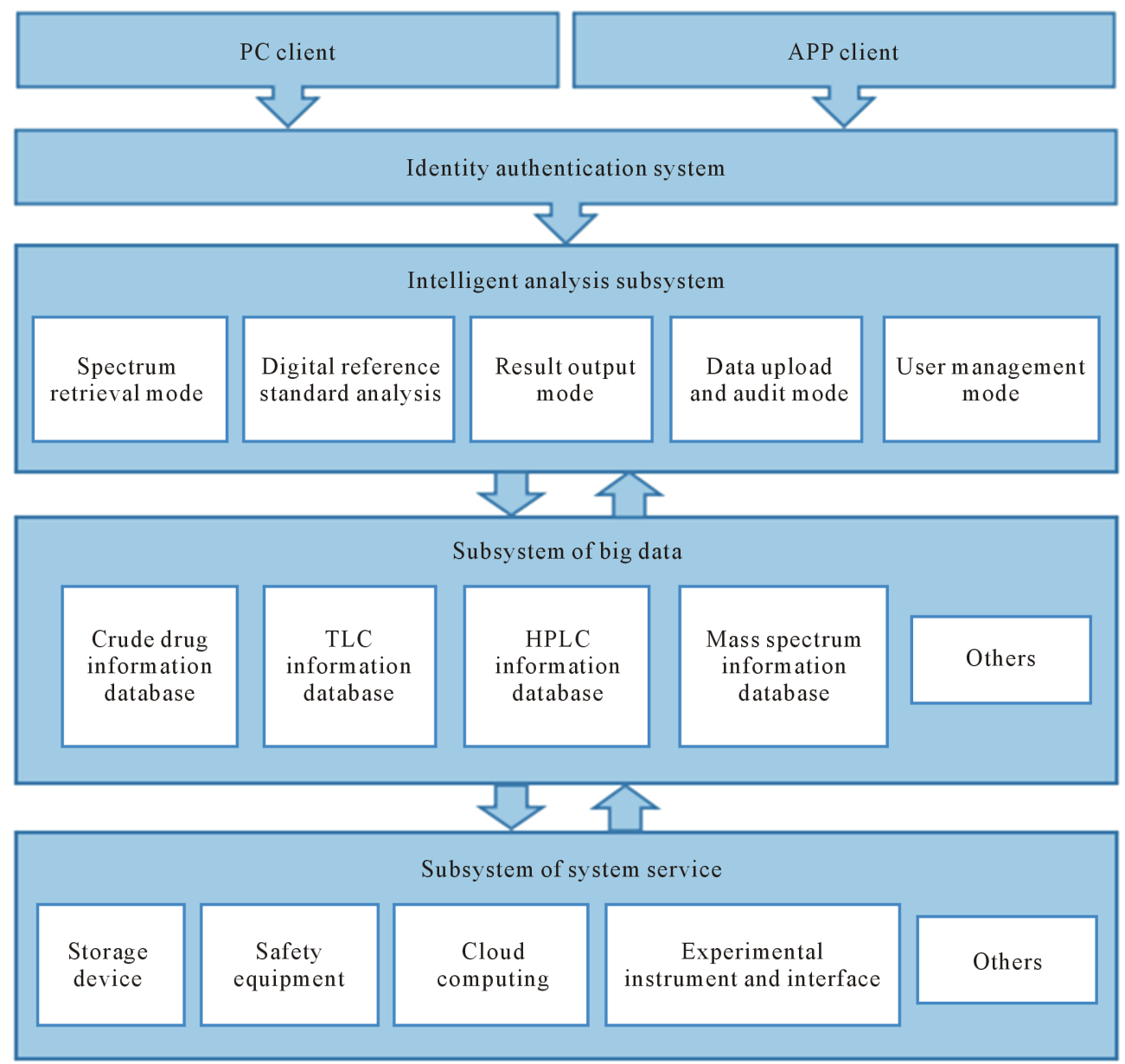

Figure 10. System design of digital reference standards. 
the database of user's input or search. Then present the details in the form of graphs. As per the needs of users, can sort and filter for the results of the hit. The subsystem uses the high throughput parallel computing method and would continuously improve and expand its application function per the user feedback.

3) Platform client subsystem. The client platform would fully take into account of the powerful data analysis and processing function of PC computer, and the flexible and convenient advantages of mobile terminal APP. Between them, the client PC is superior in terms of professional operation, and could be mainly used for data upload, analysis and processing as well as editing and auditing management functions; however, the mobile phone APP would focus on the realization of the query, retrieval, and other convenient access of the database.

4) Identity authentication system. The system is for facilitating the management and development of digital sharing service of DRS platform. Users need authorization can login DRS platform after digital authentication. After the registration, users can upload data, share data computing and database retrieval, and use other functions immediately.

The user with the identity of administrator can participate in validation, audit, and other management of uploaded data. User with unauthorized identity can achieve the function of query, browse, and part of analysis.

5) System service subsystem. The subsystem provides the system level underlying application services for the server and client for the whole DRS platform. The services include big data parallel cloud computing, cloud storage, authentication, user management, database access, analytical instruments universal data interface technology, and stoichiometry algorithm library.

\subsection{The Significance for Bringing This New Idea DRS}

It is of great improtance to put forward the new concept of DRS. On one hand, the propose of the new concept has opened up a new way of thinking to solve the RS related problems. This idea will be the origin of DRS and researchers can continue innovating and researching along this new thought and work together to establish DRS, thus breaking the bottleneck of the use of RS and bringing convenience to the holistic quality control of drugs. One the other hand, the propose of the new idea can cause more attention to the current RS using problems. Reachers will not only deal with the problems referring to the DRS idea, but also consider to find new ideas to solve the problems.

\section{Conclusion and Prospect}

Over the past two decades, information technology and the internet had a huge impact on human society. It is a major technological revolution after the industrial revolution. With the rapid development of information technology, pharmaceutical analysis changed from the classic analysis (chemical analysis) into the era of instrument analysis, and development of RS has entered a new stage combined with information technology. The appearance and development of relevant software and electronic databases of drug analysis make physical RS and paper map become digital and sharing. This is the primary stage of digitization and intelligence of RS.

Now it is the era of internet plus, big data, artificial intelligence, and other trend are irresistible. The industry workers need to have a profound understanding of these concepts, change the mode of thinking, and actively embrace the application of these methods. For example, Professor Li Xiaogang forwarded the concept of "corrosion big data", that is to establish a standardized database to conduct data modeling and the data image characterization of results. The Institute of Botany of the Chinese Academy of Sciences cooperated with Baidu and their new technology can identify close-up pictures of 1000 kinds of common flowers, accuracy rate up to $80 \%$. Their new technology combines the massive pictures of plant image library and the deep learning technology [54]. Similarly, drug analysis workers can and must use the concept of internet plus to solve the current problems in the use of RS. Compared with traditional RS, the using of digital form of RS has many advantages. On one hand, the work of preparation and calibration of RS can be reduced, thus reducing the waste of resources, environmental pollution and economizing the cost. On the other hand, the digital form of RS can provide more comprehensive, inter-related information in a systematic, standardized form to achieve the purposes of intelligent search, big data storage and Internet sharing. DRS provides new ideas and methods for the development of RS and drug quality control.

The DRS would gradually replace the physical RS. It is not only a software, but also a ecosystem to connect user, analytical instruments, quality standard, and RS feature information.

It is the necessity for the development of pharmaceutical analysis and research for RS change from physical 
form, paper atlas, and substitute RS to electronic database, until the formation of a DRS. This is the inevitable trend of the times. However, the establishment of DRS still needs several research works, and many technical problems need to be discussed and solved. This manuscript put forward the concept, characteristics, and mode of RS for reference and discussion. Of course, in future, all the RS have become digital existence, but a physical library of RS may also be necessary.

\section{Acknowledgements}

1) Project of National 12th Five-Year “major new drug discovery” (2014ZX09304307-002).

2) The National Natural Science Foundation of China (No.81303214, 81373955).

\section{References}

[1] Hang, T.J., et al. (2011) Pharmaceutical Analysis. People’s Medical Publishing House, Beijing.

[2] Chinese Pharmacopoeia Commission (2013) Chinese Pharmacopoeia. Second Supplement of 2010 Version, 383.

[3] Chinese Pharmacopoeia Commission (2015) Chinese Pharmacopoeia. 2015 Version Fourth Chapter, 290-291+333353.

[4] Chinese Pharmacopoeia Commission (1985) Chinese Pharmacopoeia. 1985 Version. First Chapter, 1+347

[5] Chinese Pharmacopoeia Commission (2005) Chinese Pharmacopoeia. 2005 Version. First Chapter, 522.

[6] Chinese Pharmacopoeia Commission (2015) Chinese Pharmacopoeia. 2015 Version. First Chapter, 191+262.

[7] Ma, L.Y. and Ma, S.C. (2010) Development and Prospect of TCM Reference Standards. China Pharmacy, 24, 12321236.

[8] China Standardization Committee on Biological Products (2000) Chinese Requirements for Biological Products.

[9] The European Directorate for the Quality of Medicines \& Health Care (2015) EDQM Reference Standard. https://crs.edqm.eu/

[10] Williams, R.L., et al. (2006) Official USP Reference Standards: Metrology Concepts, Overview, and Scientific Issues and Opportunities. Journal of Pharmaceutical and Biomedical Analysis, 40, 3-15. http://dx.doi.org/10.1016/j.jpba.2005.07.017

[11] The United States Pharmacopieial Convention (2015) USP Reference Standard. http://www.usp.org/reference-standards

[12] Hong Kong Special Administrative Region Department of Health of the People’s Republic of China (2015) Hong Kong Chinese Materia Medica Standards, Volume 7, 14.

[13] Chinese Pharmacopoeia Commission (2010) Atlas of Infrared Spectra of Drugs. Fourth Volume, 1111.

[14] Chinese Pharmacopoeia Commission (2009) Atlas of TLC Color Pictures of Chinese Crude Drugs for Chinese Pharmacopoeia, 27.

[15] Chinese Pharmacopoeia Commission (2009) An Illustrated Handbook on Microscopic Identification of Chinese Crude Drugs for Chinese Pharmacopoeia, 53.

[16] Wang, J. (2008) The IR Spectrum Database System Research. East China Normal University, Shanghai.

[17] Xie, P.S., Ma, S.C., Tu, P.F., Wang, Z.T., Stoeger, E. and Bensky, D. (2013) The Prospect of Application of Extractive Reference Substance of Chinese Herbal Medicines. Chinese Medicine, 4, 125-136. http://dx.doi.org/10.4236/cm.2013.44016

[18] Liao, H., Li, Q., Liu, R., Liu, J. and Bi, K. (2014) Fingerprint Analysis and Multi-Ingredient Determination Using a Single Reference Standard for Saposhnikoviae Radix. Analytical Sciences, 30, 1157-1163. http://dx.doi.org/10.2116/analsci.30.1157

[19] Zhong, J.-S., Wan, J.-Z., Liu, Y.-P., Ding, W.-J., Wu, X.-F. and Xie, Z.-Y. (2014) Simultaneous HPLC Quantification of Seven Chromones in Aloe barbadensis Miller Using a Single Reference Standard. Analytical Methods, 6, 4388-4395. http://dx.doi.org/10.1039/c4ay00031e

[20] Gao, W., Wang, R., Li, D., Liu, K., Chen, J., Li, H.J., Xu, X., Li, P. and Yang, H. (2016) Comparison of Five Lonicera Flowers by Simultaneous Determination of Multi-Components with Single Reference Standard Method and Principal Component Analysis. Journal of Pharmaceutical and Biomedical Analysis, 117, 345-351.

[21] Gao, X.-Y., Jiang, Y., Lu, J.-Q. and Tu, P.-F. (2009) One Single Standard Substance for the Determination of Multiple Anthraquinone Derivatives in Rhubarb Using High-Performance Liquid Chromatography-Diode Array Detection. Journal of Chromatography A, 1216, 2118-2123. http://dx.doi.org/10.1016/j.chroma.2008.11.104 
[22] Hou, J.-J., Wu, W.-Y., Liang, J., Yang, Z., Long, H.-L., Cai, L.-Y., Fang, L., Wang, D.-D., Yao, S., Liu, X., Jiang, B.-H. and Guo, D.-A. (2014) A Single, Multi-Faceted, Enhanced Strategy to Quantify the Chromatographically Diverse Constituents in the Roots of Euphorbia kansui. Journal of Pharmaceutical and Biomedical Analysis, 88, 321330.

[23] Wu, X.X., He, J., Xu, H.R., Bi, K.S. and Li, Q. (2014) Quality Assessment of Cinnamomi Ramulus by the Simultaneous Analysis of Multiple Active Components Using High-Performance Thin-Layer Chromatography and High-Performance Liquid Chromatography. Journal of Separation Science, 37, 2490-2498. http://dx.doi.org/10.1002/jssc.201400494

[24] Zhang, Y.-W., Li, Q., Lv, C.-X., Liu, X.-J., Chen, X.-H. and Bi, K.-S. (2015) Simultaneous Determination of Four Active Components in Alisma orientale (Sam.) Juz. by HPLC-DAD Using a Single Reference Standard. Journal of Pharmaceutical Analysis, 5, 85-92. http://dx.doi.org/10.1016/j.jpha.2014.12.001

[25] Li, S.P., Qiao, C.F., Chen, Y.W., Zhao, J., Cui, X.M., Zhang, Q.W., Liu, X.M. and Hu, D.J. (2013) A Novel Strategy with Standardized Reference Extract Qualification and Single Compound Quantitative Evaluation for Quality Control of Panax notoginseng Used as a Functional Food. Journal of Chromatography A, 1313, 302-307. http://dx.doi.org/10.1016/j.chroma.2013.07.025

[26] Lu, W.Y., Niu, Y.G., Yang, H.S., Sheng, Y., Shi, H.M. and Yu, L.L. (2014) Simultaneous HPLC Quantification of Five Major Triterpene Alcohol and Sterol Ferulates in Rice Bran Oil Using a Single Reference Standard. Food Chemistry, 148, 329-334. http://dx.doi.org/10.1016/j.foodchem.2013.10.027

[27] Hou, J.J., Wu, W.Y., Da, J., Yao, S., Long, H.L., Yang, Z., Cai, L.Y., Yang, M., Liu, X., Jiang, B.H. and Guo, D.A. (2011) Ruggedness and Robustness of Conversion Factors in Method of Simultaneous Determination of Multi-Components with Single Reference Standard. Journal of Chromatography A, 1218, 5618-5627. http://dx.doi.org/10.1016/j.chroma.2011.06.058

[28] Wang, W., Ma, X., Guo, X., Zhao, M., Tu, P. and Jiang, Y. (2015) A Series of Strategies for Solving the Shortage of Reference Standards for Multi-Components Determination of Traditional Chinese Medicine, Mahoniae Caulis as a Case. Journal of Chromatography A, 1412, 100-111. http://dx.doi.org/10.1016/j.chroma.2015.08.015

[29] Yang, T.W., Zhao, C., Fan, Y., Qi, L.W. and Li, P. (2015) Design of Ultraviolet Wavelength and Standard Solution Concentrations in Relative Response Factors for Simultaneous Determination of Multi-Components with Single Reference Standard in Herbal Medicines. Journal of Pharmaceutical and Biomedical Analysis, 114, 280-287. http://dx.doi.org/10.1016/j.jpba.2015.05.028

[30] Sun, L., Jin, H.Y., Pang, Y. and Ma, S.C. (2013) Two Reference Substances for Determination of Multiple Components (I): Linear Calibration Using Two Reference Substances for Identification of Chromatographic Peaks. Chinese Journal of Pharmaceutical Analysis, 33, 1424-1430.

[31] Wei, L. and Hu, C.Q. (2008) Spectral Correlation of High-Performance Liquid Chromatography-Diode Array Detection Data from Two Independent Chromatographic Runs Peak Tracking in Pharmaceutical Impurity Profiling. Journal of Chromatography A, 1190, 141-149. http://dx.doi.org/10.1016/j.chroma.2008.02.097

[32] Wang, N., Li, C.F., Zhang, D.S. and Hu, C.Q. (2011) Establishment of an HPLC Method for the Analysis of Biapenem and Its Impurities. Journal of Chinese Pharmaceutical Sciences, 20, 171-180.

[33] Wu, L., Wu, Y.Z., Shen, H.Y., Gong, P., Cao, L.J., Wang, G.J. and Hao, H.P. (2013) Quantitative Structure-Ion Intensity Relationship Strategy to the Prediction of Absolute Levels without Authentic Standards. Analytica Chimica Acta, 794, 67-75. http://dx.doi.org/10.1016/j.aca.2013.07.034

[34] Liang, Y., Hao, H.P., Kang, A., Xie, L., Xie, T., Zheng, X., Dai, C., Wan, L.R., Sheng, L.S. and Wang, G.J. (2010) Qualitative and Quantitative Determination of Complicated Herbal Components by Liquid Chromatography Hybrid Ion Trap Time-of-Flight Mass spectrometry and a Relative Exposure Approach to Herbal Pharmacokinetics Independent of Standards. Journal of Chromatography A, 1217, 4971-4979. http://dx.doi.org/10.1016/j.chroma.2010.05.056

[35] Nara Institute of Science and Technology (2015) MassBank. http://www.massbank.jp/

[36] Max Planck Institute for Molecular Plant Physiology (2015) Golm Metabolome Database. http://gmd.mpimp-golm.mpg.de/

[37] Li, Y.P., Wang, C., Gu, L.S. and Hu, C.Q. (2008) Establishent and Application Of Column-Recommending-System of Antibioitc Drugs of Chinese Pharmacopeia 2005 Edition. Chinese Journal of Pharmaceutical Analysis, 28, 1401-1404.

[38] Sadtler Research Laboratories (2015) Sadtler Infrared Database. http://www.jetting.com.cn/Bio-Rad/Sadtler/SadtlerDB_Index.html

[39] Cui, Q., Lewis, I.A., Hegeman, A.D., et al. (2008) Metabolite Identification via the Madison Metabolomics Consortium Database. Nature Biotechnology, 26, 162-164. http://dx.doi.org/10.1038/nbt0208-162

[40] Fullbeck, M., Michalsky, E., Dunkel, M. and Preissner, R. (2006) Natural Products: Sources and Databases. Natural Product Reports, 23, 347-356. http://dx.doi.org/10.1039/b513504b 
[41] Montes-Grajales, D. and Olivero-Verbel, J. (2015) EDCs DataBank: 3D-Structure Database of Endocrine Disrupting Chemicals. Toxicology, 327, 87-94. http://dx.doi.org/10.1016/j.tox.2014.11.006

[42] National Institute of Standards and Technology (2015) NIST Mass Spectral Library. http://www.nist.gov

[43] Caspi, R., Altman, T., Dreher, K., et al. (2008) The MetaCyc Database of Metabolic Pathways and Enzymes and the BioCyc Collection of Pathway/Genome Databases. Nucleic Acids Research, 36, D623-D631.

[44] Waters (2015) Waters UNIFI Scientific Information System. http://www.waters.com/waters/zh_CN/UNIFI-Scientific-Information-System/nav.htm?cid=134801359\&locale=zh_CN

[45] Agilent Technologies (2015) Aglient Technologies GC/MS, LC/MS Chemical Synthetic Drug Storehouse. http://cn.chem.agilent.com/en-US/promotions/Pages/designer-drug-kit-CN.aspx

[46] Thermo Fisher Scientific (2015) Thermo Fisher Spectrum Database. http://www.thermo.com.cn/Category375.html

[47] SHIMADZU (2015) Metabolic Enzymes in Yeast LC/MS/MS MRM Database. http://www.shimadzu.com.cn/newsinfo_contents/5398.html

[48] SHIMADZU (2015) Smart MRM Poison Edition of Database. http://www.shimadzu.com.cn/an/gcms/smart-MRM/3544.html

[49] Hastings, J., de Matos, P., Dekker, A., et al. (2013) The ChEBI Reference Database and Ontology for Biologically Relevant Chemistry: Enhancements for 2013. Nucleic Acids Research, 4, D456-D463. http://dx.doi.org/10.1093/nar/gks1146

[50] DNP (Dictionary of Natural Products) (2015). http://dnp.chemnetbase.com

[51] King's College London (2015) CHEM-TCM: Chemical Database of Traditional Chinese Medicine. http://www.chemtcm.com/

[52] Shanghai Innovative Research Center of Traditional Chinese Medicine (2008) New Drug Oriented Development System of Chinese Medicine.

[53] Hong Kong Baptist University (2015) Chinese Medicinal Herbal Medicine Image Database. http:/library.hkbu.edu.hk/electronic/libdbs/mmd/index.html

[54] Chinese Academy of Sciences (2015) Network Science and Technology Communication Platform of Chinese Academy of Sciences. http://stu.iplant.cn/web

[55] Von Hunolstein, C., Aggerbeck, H., Andrews, N., Berbers, G., Fievet-Groyne, F., Maple, P.A.C., Ölander, R.M., Raux, M. and Tischer, A. (2000) European Sero-Epidemiology Network: Standardisation of the Results of Diphtheria Antitoxin Assays. Vaccine, 18, 3287-3296. http://dx.doi.org/10.1016/S0264-410X(00)00125-0

\section{Abbreviations}

RS, reference standards

$\mathrm{RM}$, reference materials

CRS, chemical reference standards

HRS, herbal reference standards

ERS, extractive reference standards

BRS, biological reference standards

DRS, digital reference standards

TCM, Traditional Chinese Medicines

PC, pharmaceutical chemicals

$\mathrm{PE}$, pharmaceutical excipients 